\title{
Nasal Septum
}

National Cancer Institute

\section{Source}

National Cancer Institute. Nasal Septum. NCI Thesaurus. Code C33160.

The thin wall between the two nasal cavities. 